\title{
A Different and Realistic Approach to Inter Base Conversion for Number System
}

\author{
Saurabh Rawat \\ Dept. of Computer Science \& \\ Engg. \\ Graphic Era University \\ Dehradun, India
}

\author{
Bhaskar Nautiyal \\ Dept. of Computer Science \& \\ Engg. \\ Graphic Era University \\ Dehradun, India
}

\author{
Anushree Sah \\ University of Greenwich, \\ London, U.K
}

\begin{abstract}
A number system (or system of numeration) is a writing system for expressing numbers, that is a mathematical notation for representing numbers of a given set, using digits or other symbols in a consistent manner. It can be seen as the context that allows the symbols "11" to be interpreted as the binary symbol for three, the decimal symbol for eleven, or a symbol for other numbers in different bases. A number system is a set of rules and symbols used to represent a number. Binary $(0,1)$ and other famous number systems, octal ( 0-7 ), hexadecimal ( 0-15) are based on same fundamental concept of decimal number system ( 0-9). The knowledge of number systems, their representation, limits, arithmetic compliments and inter conversion of numbers between prescribed number systems is essential for understanding of computers and successful programming for digital devices. Understanding all these number conversions ( from one base to decimal and to another base ) and related concepts requires a lot of time and large time consuming techniques to expertise. In this paper we have elaborated concepts of conversion among different bases and proposed with the help of a table to obtain simply and effectively solution from one base to another base conversion, without converting to decimal number system. This effort will also enhance the knowledge intellectuals understanding and practicing of number system conversions.
\end{abstract}

\section{General Terms}

Number system, binary,octal, hexadecimal,inter conversions

\section{Keywords}

Number system, binary,octal, hexadecimal,inter conversions

\section{INTRODUCTION}

A number system defines a set of values to represent quantity. We talk about the number of people attending a class, the number of modules taken by each student and use numbers to represent grade.[2]

Number System can be categorized in two systems:-

(a) Non-Positional Number System

(b) Positional Number System

Non-Positional Number System- In ancient times, people used to count on fingers, when the fingers became insufficient for counting, stones, pebbles or sticks were used to indicate values. But it was very difficult to perform arithmetic with such a number system as there is no symbol for zero.

Positional Number System- In this system the value of each digit is defined not by the symbol but also by the symbol position. Positional Number System is used to perform arithmetic. Existing Positional number system is decimal number system. Apart from the decimal number system, there are binary number system, octal number system and hexadecimal number system.

Base (Radix)- In the number system the base or radix tells the number of symbols used in the system. In the earlier days, different civilizations were using different radixes. The Egyptian used the radix 2, the Babylonians used the radix 60 and Mayans used 18 and 20.

The base of a number system is indicated by a subscript (decimal number) and this will be followed by the value of the number. For example $(879)_{10},(457)_{8}$ and $(56 A)_{16}$. Beside the fact that many students know the decimal (base 10) system, and are very comfortable with performing operations using this number system, it is too important for students to know and understand that the decimal system is not the only number system. By studying other number systems such as binary (base 2) quaternary (base 4), senary (base 6), octal (base 8), unodecimal ( base 11 ), duodecimal ( base 12 ), tridecimal ( base 13 ), quadrodecimal (base 14 ), pentadecimal (base 15 ), hexadecimal (base 16) and so forth [3], students will gain a better understanding of how number systems work in general. It is well known that the design of computers begins with the choice of number system, which determines many technical characteristics of computers. In modern computer, number system used is binary number system. All other number systems are converted to binary number system for computer to access data.

\subsection{Digits and their positions}

Such a symbol used in a system of numeration or one of the ten Arabic number symbols, 0 through 9 is called digit. The first digit of/in any number system is always a zero. For example, a base 2 (binary) numbers have 2 digits: 0 and 1, a base 8 (octal) numbers have 8 digits: 0 through 7 and so forth. Remember that a base 10 or decimal numbers does not contain the digit 10 , similarly base 8 or octal numbers does not contain a digit 8 , and same is the case for the other number systems. Once the digits of a number system are understood, each and every larger numbers can be constructed using positional notation or place-value notation method. According to this method, the first right most digit (integer) has a unit's position in decimal number. Further, to the left of the units position is the ten's position, the position to the left of the ten's position is the hundred's position and so forth. Here, the units position has a weight of $1 \mathbf{O}^{\mathbf{O}}$, or 1 ; the tens position has a weight of $\mathbf{1 0}^{1}$, or 10 ; and the hundreds position has a weight of $\mathbf{1 \mathbf { O } ^ { 2 }}$, or 100 . The exponential powers of the positions are significant for understanding numbers in other number systems. Always, the unit's position in any number system is the position to the left of the radix point. For example the position to the left of the binary (radix) 
point is always $2^{\circ}$, or 1 ; the position to the left of the octal (radix) point is always $8^{\circ}$, or 1 and so on. Similarly the position to the left of the unit's position is always the number whose base is raised to the first power; i.e. $2^{1}, 8^{1}$ and so on. These concepts can be extended to each and every number system.

\subsection{Representation of number in any base system}

A number in any base system can be represented in a generalized format as follows: $N=a_{n} b^{n}+a_{n-1} b^{n-1}+----+a_{1} b^{1}+a_{0} b^{0}$ where $\mathrm{N}=$ Number, $\mathrm{b}=$ Base, $\mathrm{a}=$ any digit in that base

For example number 142 can be represented in various number systems as follows:

Table 1. Number representations in various number systems

\begin{tabular}{|c|l|l|l|}
\hline Decimal & 142 & $1 \times 10^{2}+4 \times 10^{1}+2 \times 10^{0}=100+40+2$ & 142 \\
\hline Binary & $\begin{array}{l}100 \\
011 \\
10\end{array}$ & $\begin{array}{l}1 \times 2^{7}+0 \times 2^{6}+----+1 \times 2^{3}+1 \times 2^{2}+1 \times 2^{0}= \\
128+0+0+0+8+4+2+0\end{array}$ & 142 \\
\hline Octal & 216 & $2 \times 8^{2}+1 \times 8^{1}+6 \times 8^{0}=128+8+6$ & 142 \\
\hline $\begin{array}{c}\text { Hexa- } \\
\text { decimal }\end{array}$ & $8 \mathrm{E}$ & $8 \times 16^{1}+E \times 16^{0}=128+4$ & 142 \\
\hline
\end{tabular}

\subsection{Decimal Number System}

The decimal number system is known as international system of numbers [8]. It is also called base 10 or denary number system. It uses 10 as its base. It is the numerical base most widely used by modern civilization [7].

Decimal notation is a base-10 positional notation system. Positional decimal systems include a zero and use symbols (called digits) for the ten values i.e. $0,1,2,3,4,5,6,7,8$, and 9 to represent any number, no matter how large or how small.

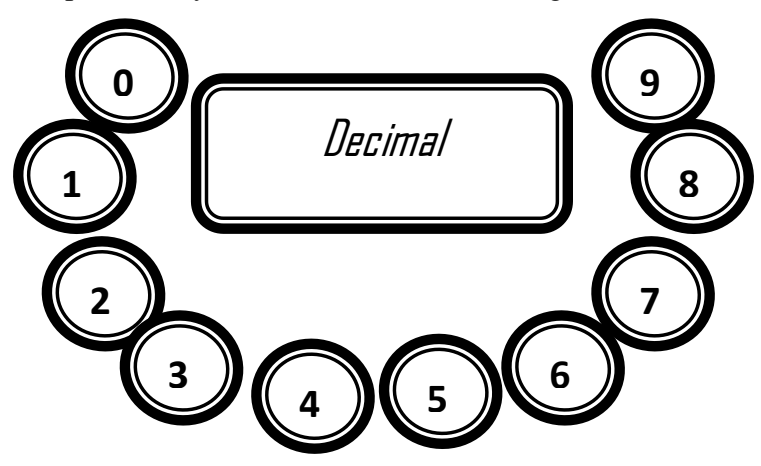

Fig 1: Symbols used in decimal number system
Let's examine the decimal value of 365.5 . You know that this value is three hundred sixty five and one-half. Now examine the position of each number:

$365.5 * .5$ represents 0.5 or $1 / 2$ units

$$
\begin{aligned}
& \text { * } \quad 5 \text { represent } 5 \text { units } \\
& \text { * } \quad 6 \text { represent } 60 \text { units, and } \\
& \text { * } \quad 3 \text { represent } 300 \text { units }
\end{aligned}
$$

Each digit has its own value (weight) as shown in the above example. Now let's look at the value of the base 10 number 365.5 with the positional notation line graph:

Decimal point

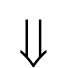

$$
\begin{aligned}
& 10^{2} 10^{1} 10^{0} \cdot 10^{-1} \\
& \begin{array}{lllll}
3 & 6 & 5 & 5
\end{array} \\
& 10^{2}=3 \times 100, \text { or } 300 \\
& 10^{1}=6 \times 10, \operatorname{or} 60 \\
& 10^{0}=5 \times 1 \text { or } 5 \\
& 10^{-1}=5 \times .1 \text { or. } 5
\end{aligned}
$$

We can see that the power of the base is multiplied by the number in that position to determine the value for that position. All numbers to the left of the decimal point are whole numbers or integers, and all numbers to the right of the decimal point are fractions.

\subsection{Binary Number System}

The number system with base 2 is known as the binary number system. Only two symbols are used to represent numbers in this system i.e. 0 and 1 known as bits. It is a positional system i.e. every position is assigned a specific weight. Moreover, its number has two parts the Integral part or integers and the fractional part or fractions, set a part by a radix point. For example (1101.101)2

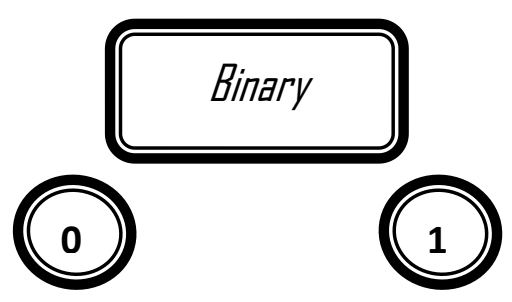

Fig 2: Symbols used in binary number system

In the binary number system the left-most bit is known as most significant bit (MSB) and the right-most bit is known as the least significant bit (LSB). The following graph shows the position and the power of the base ( 2 in this case): 


\section{$\ldots . .2^{3} 2^{2} 2^{1} 2^{0} \cdot 2^{-1} 2^{-2} 2^{-3} \ldots$}

The arithmetic operations such as addition, subtraction, multiplication and division of decimal numbers are also performed on binary numbers. Also binary arithmetic is much simpler than decimal arithmetic because only two digits, 0 and 1 are used here.

\subsection{Octal Number System}

Octal stands for 8 , so the number system with base 8 is known as the octal number system. This system uses eight symbols, $0,1,2,3,4,5,6$, and 7 to represent the number. Hence, any octal number can not have any digit greater than 7 [10].

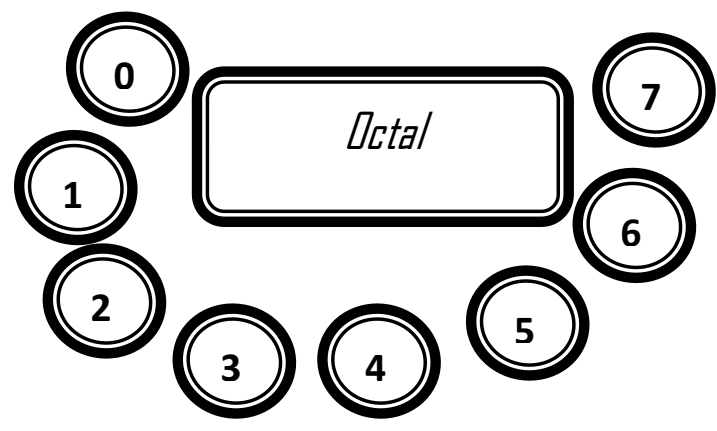

Fig 3: Symbols used in octal number system

Similar to decimal and binary number systems, it is also a positional system; the octal number system uses power of 8 to determine the value of a number's position. The following graph shows the positions and the power of the base ( 8 in this case):

$$
\ldots 8^{3} 8^{2} 8^{1} 8^{0} .8^{-1} 8^{-2} 8^{-3} \ldots
$$

Integral and fractional part of octal number is set a part (separated) by a radix point, for example (6522. 3712)8. In digital transmission system it is highly tedious to handle long strings of binary numbers. It may also cause errors. Therefore, octal numbers are useful to use for entering binary data and displaying certain information in short.

\subsection{Hexadecimal Numbering System}

Hexadecimal number system is very popular in computer uses. The base for hexadecimal number system is 16 which require 16 distinct symbols to represent the number. These are numerals 0 through 9 and alphabets A through $F$ [11]. This is an alphanumeric number system because its uses both alphabets and numerical to represent a hexadecimal number. Hexadecimal number system use $0,1,2,3,4,5,6,7,8,9$, A, $\mathrm{B}, \mathrm{C}, \mathrm{D}, \mathrm{E}$, and $\mathrm{F}$.

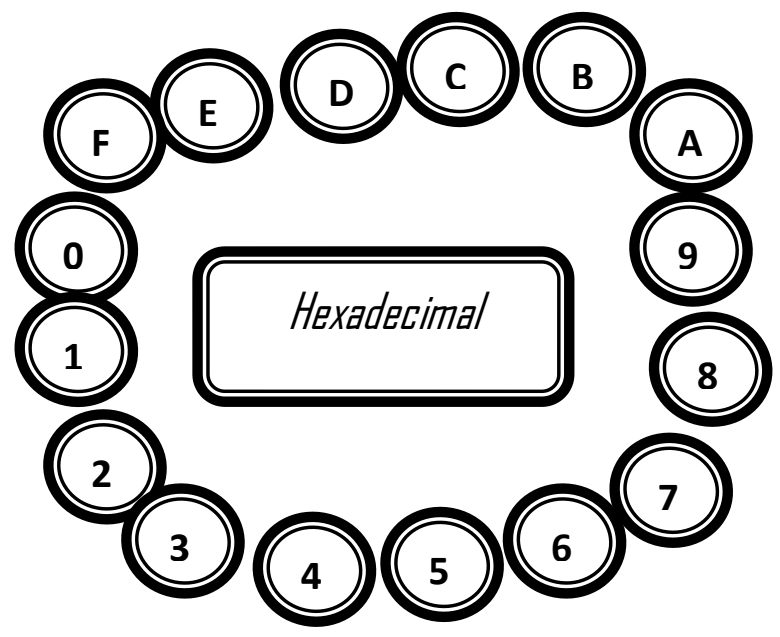

Fig 4: Symbols and alphabets used in hexadecimal number system

Any number in hexadecimal number system having integral and fractional parts can be represented as (E45.673)16.

$$
\ldots 16^{3} 16^{2} 16^{1} 16^{0} .16^{-1} 16^{-2} 16^{-3} \ldots
$$

Like the binary, octal, and decimal systems, the hexadecimal number system is a positional system. Powers of 16 are used for the positional values of a number. The following graph shows the positions and power of the base (16 in this case):

\section{ARITHMETIC OF NUMBER SYSTEMS}

The arithmetic is the most basic branch of mathematics, used by almost everyone from simple day-to-day counting to advanced science and business calculations. It simply refers to the basic mathematical operation such addition, subtraction, multiplication and division. As at the present, binary number system is the most common number system used by computer systems. However, long ago, there were such computer systems which were based on the decimal (base 10) number system rather than the binary number system. Therefore, despite the truth that decimal arithmetic is generally inferior to binary arithmetic; the need for decimal arithmetic still persists. Remember that the arithmetic operations such as addition, subtraction, multiplication and division of decimal numbers can be performed on all other numbers from binary, octal and hexadecimal as well. Therefore, in this section of the paper only the decimal arithmetic is described. 


\subsection{Decimal arithmetic [2]}

Addition: In decimal numbers addition, a one quantity is added to another (for example $5+7=12$ ). The basic terms of addition are: AUGEND: The quantity to which an addend is added (first number i.e. 5 in this example) ADDEND: A number to be added to an earlier number (second number i.e. 7 in this example) SUM: The result of an addition (i.e. 12, the sum of 5 and 7) CARRY: A carry is produced when the sum of two or more digits equals or exceeds the base of the number system in use. The following table shows the addition operation of four number systems with the help of example from each system;

Table2. Addition in various number systems

\begin{tabular}{|l|l|}
\hline Binary & $(1000)_{2}+(0111)_{2}=(1111)_{2}$ \\
Octal & $(75)_{8}+(32)_{8}=(127)_{8}$ \\
Decimal & $\left((92)_{10}+(24)_{10}=(116)_{10}\right.$ \\
Hexadecimal & $(1 A)_{16}+(24)_{16}=(3 E)_{16}$ \\
\hline
\end{tabular}

Subtraction: Subtraction is the opposite of addition. Subtraction finds the difference between two numbers, the minuend minus the subtrahend. In other words, subtraction means to take away a part from the whole number or one number from another number. If the minuend is larger than the subtrahend, the difference is positive; if the minuend is smaller than the subtrahend, the difference is negative; if they are equal, the difference is zero. For example: $25-7=18$ The basic terms of subtraction are: MINUEND: The number from which another number is to be subtracted (i.e. 25 in the above example) SUBTRAHEND: The number to be subtracted (i.e. 7 here) REMAINDER or DIFFERENCE: That number which is left after subtraction (i.e. 18 here) BORROW: To transfer a digit (equal to the base number) from the next higher order column for the purpose of subtraction. The following table shows the subtraction operation of four number systems with the help of example from each system;

Table 3.Subtraction in various number systems

\begin{tabular}{|ll|}
\hline Binary & $(1001)_{2}-(011)_{2}=(110)_{2}$ \\
Octal & $(74)_{8}-(24)_{8}=(50)_{8}$ \\
Decimal & $(94)_{10}-(27)_{10}=(67)_{10}$ \\
Hexadecimal & \\
& $\left((3 F)_{16}-(09)_{16}=(36)_{16}\right.$ \\
\hline
\end{tabular}

Multiplication: Multiplication is also one of the basic operations of arithmetic. It also combines two numbers into a single number, called "product". In this arithmetic operation simply multiply the multiplicand by each digit of the multiplier and then add up all the properly shifted results. For example: $32 \times 8$ The basic terms of multiplication are: MULTIPLIER: The number by which another number is multiplied (i.e. 8 is the multiplier in above example

MULTIPLICAND: The number that is to be multiplied by another. Here the multiplicand is 32 PRODUCT: The number or quantity obtained by multiplying two or more numbers together, i.e. $32 \times 8=256$ Binary, octal, and hexadecimal multiplication is similar to decimal multiplication except that base and counting is changed accordingly. Each digit of the multiplier (2nd number), multiplies to the whole multiplicand number (1st number). The following table shows the multiplication operation of four number systems with the help of example from each system;

Table4. Multiplication in various number systems

\begin{tabular}{|ll|}
\hline Binary & $(1001)_{2} \times(011)_{2}=(11011)_{2}$ \\
Octal & $(74)_{8} \times(24)_{8}=(2260)_{8}$ \\
Decimal & $(82)_{10} \times(07)_{10}=(574)_{10}$ \\
& $(3 A)_{16} \times(07)_{16}=(196)_{16}$
\end{tabular}

Division: Division is basically the opposite of multiplication. Division obtains the quotient of two numbers, when the dividend is divided by the divisor. Any dividend divided by zero is undefined. If the dividend is larger than the divisor, the quotient is greater than 1 otherwise it is less than 1 . While in reverse if the quotient is multiplied by the divisor, it always yields the dividend. For example: $45 \div 3=15$ The basic terms of division are: DIVIDER: One number that divides another number (i.e. 3 here) DIVIDEND: A number to be divided (i.e. 45 here) QUOTIENT: The number obtained by dividing one number by another (i.e. 15) REMAINDER: The number left over when one number is divided by another (in this example remainder is 0) Binary, octal, and hexadecimal division is obtained using the same procedure like decimal division except that base and counting is changed accordingly. The following table shows the division operation of four number systems with the help of example from each system;

Table 5. Division in various number systems

\begin{tabular}{|ll|}
\hline Binary & $(1001)_{2} /(011)_{2}=(11)_{2}$ \\
Octal & $(74)_{8} /(24)_{8}=(03)_{8}$ \\
Decimal & $(72)_{10} /(08)_{10}=(09)_{10}$ \\
\hline
\end{tabular}




$(3 F)_{16} /(09)_{16}=(07)_{16}$
Hexadecimal

\section{INTER CONVERSION}

As discussed earlier, the most common and well-known number systems are the decimal, binary, octal and hexadecimal. Number systems are given in the ascending order as,

Binary

Octal

Decimal

Hexadecimal

A given number in any of the above number systems may consist of two parts i.e. the integral part and the fractional part. Each part some times, required a different technique for conversion. In other words, in case of fractions the conversion process requires additional techniques. So as a whole, more than 20 steps and various techniques are required to complete the conversion process.

It is very difficult to cover all the conversion in short time of one contact hour or so. To overcome this problem a table is given as under which reveals the conversion between the four number systems in three steps along the methods of conversion (for integers and fractions). Remember, in step 2 and 3 conversion technique (method) for integral and fractional part is not mentioned in the table. It is so, because in both steps, same technique is used for both integers and fractions conversion.
Table 6. Conversion table for Decimal, Binary, Octal and Hexadecimal along the conversion techniques [2]

\begin{tabular}{|c|c|c|}
\hline $\begin{array}{l}\text { Step } \\
\text { No: }\end{array}$ & Part-A & Part-B \\
\hline Step1 & $\begin{array}{l}\text { Decimal - to - others } \\
\text { [binary, octal, } \\
\text { hexadecimal] }(==) 10 \\
\Rightarrow(==) 2,8,16 \text { Integer: } \\
\text { repeated division } \\
\text { method Fraction: } \\
\text { repeated multiplication } \\
\text { method }\end{array}$ & $\begin{array}{l}\text { Others [binary, octal, } \\
\text { hexadecimal] - to - } \\
\text { decimal (==)2,8,16 } \\
\Rightarrow(==) 10 \text { Integer: } \\
\text { sum of [(+ve weights }) \times \\
\text { (integer)] Fraction: sum } \\
\text { of[(-ve weights }) \times \\
(\text { fraction })]\end{array}$ \\
\hline Step2 & $\begin{array}{l}\text { binary - to - other } \\
\text { [octal, hexadecimal] } \\
(==) 2 \Rightarrow{ }_{(==) 8,16} \text { To } \\
\text { octal: replace group of } \\
\text { 3-binary bits by octal } \\
\text { digit To hex: replace } \\
\text { group of 4-binary bits } \\
\text { by hexadecimal digit } \\
\text { (same method for both } \\
\text { integral and fraction } \\
\text { part) }\end{array}$ & $\begin{array}{l}\text { Other [octal, } \\
\text { hexadecimal] - to - } \\
\text { binary (==)8,16 } \Rightarrow \\
(==) 2 \text { From octal: } \\
\text { replace each octal digit } \\
\text { by 3-bit binary From } \\
\text { hex: replace each hexa- } \\
\text { decimal digit by 4-bit } \\
\text { binary (same method } \\
\text { for both integral and } \\
\text { fraction part) }\end{array}$ \\
\hline Step3 & $\begin{array}{l}\text { octal to hexadecimal } \\
(==) 8 \Rightarrow(==) 16 \\
\text { Direct conversion not } \\
\text { applicable Octal } \Rightarrow \\
\text { Decimal } \Rightarrow \\
\text { Hexadecimal }\end{array}$ & $\begin{array}{l}\text { hexadecimal to octal } \\
(==) 16 \Rightarrow(==) 8 \\
\text { Direct conversion not } \\
\text { applicable Hexadecimal } \\
\Rightarrow \text { Decimal } \Rightarrow \text { Octal }\end{array}$ \\
\hline
\end{tabular}

\section{Inter conversion Base Tabular Method}

Through this table we propose a different approach towards inter base conversion. To convert a number from one base to another table can be directly used and developed further on. With the help of table we can easily see conversions from one base to another, without converting to decimal. This will we suppose save time, and directly we can go for conversions. After going through the table it can easily make out that in table, numbers follow a definite pattern, filled with different colors to recognize easily. 
Table 7. Inter Base conversion table

\begin{tabular}{|c|c|c|c|c|c|c|c|c|c|c|c|c|c|c|}
\hline N/B & 2 & 3 & 4 & 5 & 6 & 7 & 8 & 9 & 10 & 11 & 12 & 13 & 14 & 15 \\
\hline 1 & 1 & 1 & 1 & 1 & 1 & 1 & 1 & 1 & 1 & 1 & 1 & 1 & 1 & 1 \\
\hline 2 & 10 & 2 & 2 & 2 & 2 & 2 & 2 & 2 & 2 & 2 & 2 & 2 & 2 & 2 \\
\hline 3 & 11 & 10 & 3 & 3 & 3 & 3 & 3 & 3 & 3 & 3 & 3 & 3 & 3 & 3 \\
\hline 4 & 100 & 11 & 10 & 4 & 4 & 4 & 4 & 4 & 4 & 4 & 4 & 4 & 4 & 4 \\
\hline 5 & 101 & 12 & 11 & 10 & 5 & 5 & 5 & 5 & 5 & 5 & 5 & 5 & 5 & 5 \\
\hline 6 & 110 & 20 & 12 & 11 & 10 & 6 & 6 & 6 & 6 & 6 & 6 & 6 & 6 & 6 \\
\hline 7 & 111 & 21 & 13 & 12 & 11 & 10 & 7 & 7 & 7 & 7 & 7 & 7 & 7 & 7 \\
\hline 8 & 1000 & 22 & 20 & 13 & 12 & 11 & 10 & 8 & 8 & 8 & 8 & 8 & 8 & 8 \\
\hline 9 & 1001 & 100 & 21 & 14 & 13 & 12 & 11 & 10 & 9 & 9 & 9 & 9 & 9 & 9 \\
\hline 10 & 1010 & 101 & 22 & 20 & 14 & 13 & 12 & 11 & 10 & 10 & 10 & 10 & 10 & 10 \\
\hline 11 & 1011 & 102 & 23 & 21 & 15 & 14 & 13 & 12 & 11 & 10 & 11 & 11 & 11 & 11 \\
\hline 12 & 1100 & 110 & 30 & 22 & 20 & 15 & 14 & 13 & 12 & 11 & 10 & 12 & 12 & 12 \\
\hline 13 & 1101 & 111 & 31 & 23 & 21 & 16 & 15 & 14 & 13 & 12 & 11 & 10 & 13 & 13 \\
\hline 14 & 1110 & 112 & 32 & 24 & 22 & 20 & 16 & 15 & 14 & 13 & 12 & 11 & 10 & 14 \\
\hline 15 & 1111 & 120 & 33 & 30 & 23 & 21 & 17 & 16 & 15 & 14 & 13 & 12 & 11 & 10 \\
\hline 16 & 10000 & 121 & 100 & 31 & 24 & 22 & 20 & 17 & 16 & 15 & 14 & 13 & 12 & 11 \\
\hline 17 & 10001 & 122 & 101 & 32 & 25 & 23 & 21 & 18 & 17 & 16 & 15 & 14 & 13 & 12 \\
\hline 18 & 10010 & 200 & 102 & 33 & 30 & 24 & 22 & 20 & 18 & 17 & 16 & 15 & 14 & 13 \\
\hline 19 & 10011 & 201 & 103 & 34 & 31 & 25 & 23 & 21 & 19 & 18 & 17 & 16 & 15 & 14 \\
\hline 20 & 10100 & 202 & 110 & 40 & 32 & 26 & 24 & 22 & 20 & 19 & 18 & 17 & 16 & 15 \\
\hline 21 & 10101 & 210 & 111 & 41 & 33 & 30 & 25 & 23 & 21 & $1(10)$ & 19 & 18 & 17 & 16 \\
\hline 22 & 10110 & 211 & 112 & 42 & 34 & 31 & 26 & 24 & 22 & 20 & $1(10)$ & 19 & 18 & 17 \\
\hline 23 & 10111 & 212 & 113 & 43 & 35 & 32 & 27 & 25 & 23 & 21 & $1(11)$ & $1(10)$ & 19 & 18 \\
\hline 24 & 11000 & 220 & 120 & 44 & 40 & 33 & 30 & 26 & 24 & 22 & 20 & $1(11)$ & $1(10)$ & 19 \\
\hline 25 & 11001 & 221 & 121 & 100 & 41 & 34 & 31 & 27 & 25 & 23 & 21 & $1(12)$ & $1(11)$ & $1(10)$ \\
\hline 26 & 11010 & 222 & 122 & 101 & 42 & 35 & 32 & 28 & 26 & 24 & 22 & 20 & $1(12)$ & 1(11) \\
\hline 27 & 11011 & 1000 & 123 & 102 & 43 & 36 & 33 & 30 & 27 & 25 & 23 & 21 & $1(13)$ & $1(12)$ \\
\hline 28 & 11100 & 1001 & 130 & 103 & 44 & 40 & 34 & 31 & 28 & 26 & 24 & 22 & 20 & $1(13)$ \\
\hline 29 & 11101 & 1002 & 131 & 104 & 45 & 41 & 35 & 32 & 29 & 27 & 25 & 23 & 21 & $1(14)$ \\
\hline 30 & 11110 & 1010 & 132 & 110 & 50 & 42 & 36 & 33 & 30 & 28 & 26 & 24 & 22 & 20 \\
\hline
\end{tabular}


a) Observe the base conversion table carefully.

b) It follows a unique pattern as we move from one base to another in the table.

( 1 ), ( 1,2$),(1,2,3),(1,2,3,4)$. and so on

c) $(10,11),(10,11,12),(10,11,12,13),(10,11,12,13,14) \ldots$ and so on.

d) $(20,21,22),(20,21,22,23),($ 20, 21,22 23,24 $) \ldots \ldots \ldots \ldots$ and so on.

e) As we follow the pattern ( 1 ), ( 1,2 ), ( 1,2,3 ), (1,2,3,4) ............., there is a no shift adjacently of values from one base to another. (the value of number in adjacent base is same).

f) Following the pattern $(10,11),(10,11,12),(10,11,12,13)$, $(10,11,12,13,14) \ldots \ldots \ldots \ldots \ldots$, there is a shift of one row downward adjacently from one base to another. (The value of number in adjacent base is one less than the previous one)

g) With pattern $(20,21,22),(20,21,22,23),(20,21,22$ $23,24) \ldots . . . \ldots$...shift is of two row adjacently from one base to another. . ( the value of number in adjacent base is two less than the previous one ).

h) Similarly with $(30,31,32,33),(30,31,32,33,34) \ldots \ldots \ldots . . . i t$ is of three row downward adjacently(the value of number in adjacent base is three less than the previous one ).

\section{Case 1}

Table 8. Base conversion

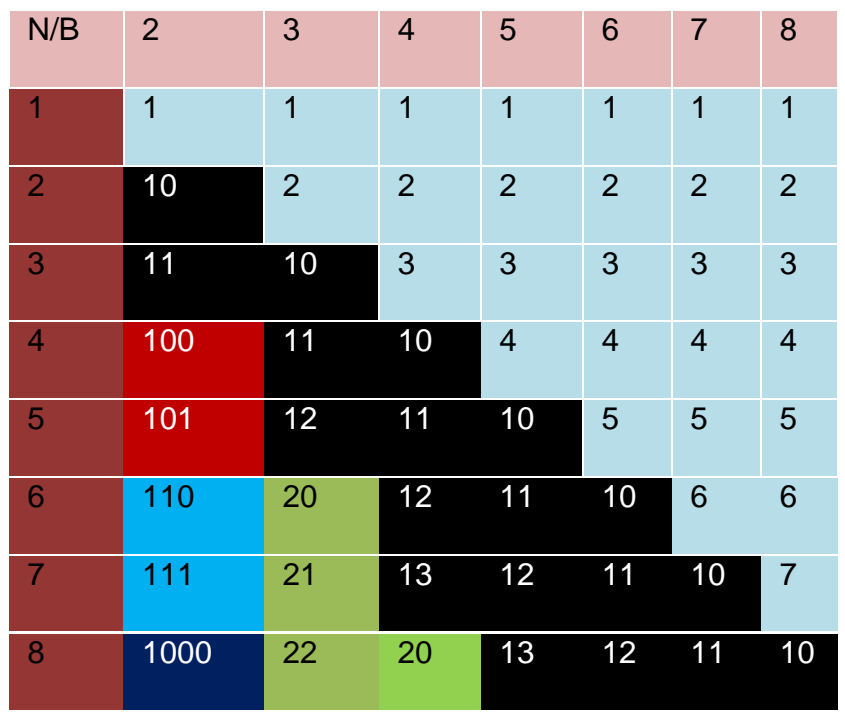

To convert a number from base 4 to base 5 .

Decimal number 7 in base 4 is

$$
=(13)_{4}
$$

\section{To convert it into base 5}

\section{Conventional method----}

$$
\begin{aligned}
& (13)_{4}=1 \times 4^{1}+3 \times 4^{0}=(7)_{10} \\
& 7 / 5=1+\text { remainder } 2=(12)_{5}
\end{aligned}
$$

Tabular Method

Decimal number 7 from table in base 4 is

$$
=(13)_{4}
$$

As it is with the pattern $(10,11),(10,11,12)$, $(10,11,12,13) \ldots \ldots \ldots .$. and so on

13 shifts one row downward adjacently, and value of number in next base , that is base 5 would be one less $=12$ as also seen from the table.

So in base 5 decimal number 7 is

$$
=(12)_{5}
$$

Further in base 6,it would be

$$
=(11)_{6}
$$

And in base 7 it is

$$
=(10)_{7}
$$

Case 2:

Table 9. Base conversion ( $N=$ Number, $B=B a s e)$

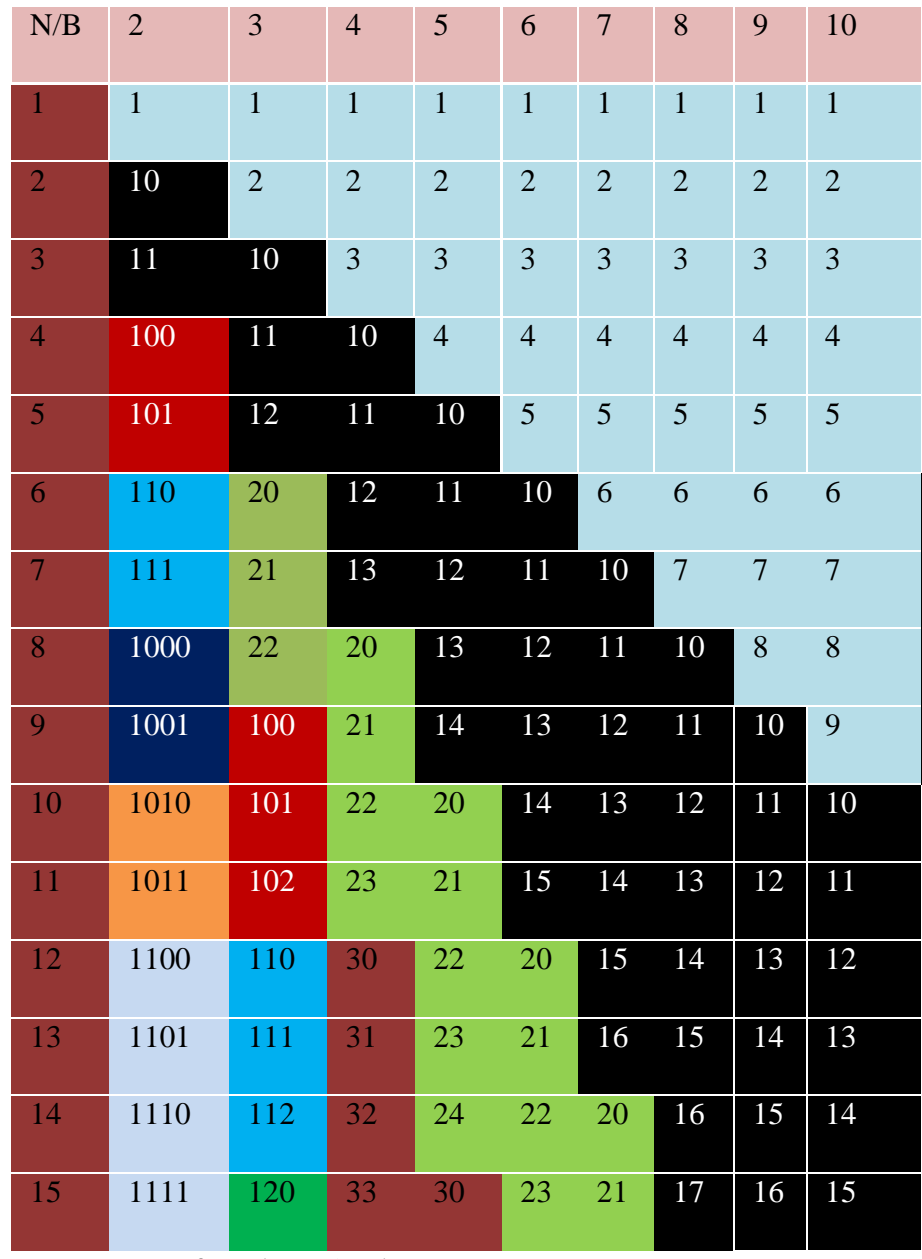

To convert from base 5 to base 7

Decimal number 14 from base 5 to base 7 . 
Conventional method.........

In base $5,14=(24)_{5}$

$(24)_{5}=2 \times 5^{1}+4 \times 5^{0}=(14)_{10}$

$14 / 7=2+$ remainder $0,=(20)_{7}$

\section{Tabular Method}

As it follows the pattern $(20,21,22),(20,21,22,23) \ldots \ldots \ldots .$. and so on it has downward shift of two, 24 shifts two places downward adjacently. And the value in the next base reduces by two, that is 24 in base 5 is 22 in next adjacent base.

In base 5 decimal number 14 is $\quad=(24)_{5}$

In base 6 it would be $\quad=(22)_{6}$

And in base 7 it is $=(20)_{7}$

\section{Case 3}

Refer to the main table above

To convert from base 10 to base 12 .

Decimal number 25 from base 10 to base 12

Conventional method

In base $10,25=(25)_{10}$

$(25)_{10}=2 \times 10^{1}+5 \times 10^{0}=(25)_{10}$

$25 / 12=2+$ remainder $1,=(21)_{12}$

\section{Tabular Method}

As it follows the pattern $(20,21,22)$, ( 20,21,22,23 ), ...........so on, it has downward shift of two places vertically adjacently and further two places in next immediate base.

And value in next base reduces by two, that is 25 in base 10 , 23 in next base 11 and further 21 in next adjacent base.

In base 10 decimal number $25,=(25)_{10}$

In base 11 decimal number $25, \quad=(23)_{11}$

In base 12 decimal number $25 \quad=(21)_{12}$

So with the table we can directly calculate the conversions without converting to decimal.

\section{Conclusion and further work}

Here we have elaborated the concepts of number systems and particularly laid emphasis on inter conversion base system. We have introduced a unique concept of converting from one base to another by using tabular method. Tabular method is quite simple, effective and finally less time consuming. Direct conversions can be achieved by studying the table and the unique pattern the number follows. Due to benefits of consuming less time in inter base conversions, which include greater speed of arithmetic operations realization, greater density of memorized information, better usage of transmission paths and decreasing of pin numbers of integrated circuits, this paper concludes that inter base conversions through tabular method remain a viable field of research and a promising future for the replacement of conventional inter base conversions. This study will be very helpful for researchers and intellectuals to easy understanding and practicing of number systems in the field of computer science and technology.

\section{REFERENCES}

[1] Shahid Latif, Asad Malook, Fahad Masoud, Muhammad Sohaib, "Proposed NS-Calculator for Well-known Number Systems ", International Journal of Computer Applications (0975-8887), Volume32- no 1, October 2011

[2] Shahid Latif, Junaid Qayyum, Muhammad Lal, Faheem Khan "Complete description of well-known number systems using single table" International Journal of Engineering and Computer Science (IJECS-IJENS), Volume 11, Issue 03

[3] Debasis Das , Dr U A Lanjewar, ' Realistic Approach of Strange Number System from Unodecimal to Vigesimal ", International Journal of Computer Science and Telecommunications, Volume 3, issue 1, January 2012.

[4] Ramesh S. Gaonkar Microprocessor Architecture, Programming, and Applications with the 8085/8080A Published by H.S. Poplai for Wiley Eastern Limited, New Delhi ISBN: 0852262973

[5] Davenport, Harold, The Higher Arithmetic:An Introduction to the Theory of Numbers (7th ed.), Cambridge University Press, Cambridge, UK,1999, ISBN 0-521-63446-6

[6] Histoire universelle des chiffres, Georges Ifrah, Robert Laffont, 1994 (Also:The Universal History of Numbers: From prehistory to the invention of the computer, Georges Ifrah, ISBN 0471393401, John Wiley and Sons Inc., New York, 2000. Translated from the French by David Bellos, E.F. Harding, Sophie Wood and Ian Monk)

[7] M. MORRIS MANO "Digital Logic and Computer Design" 2nd edition

[8] D. Nasib S. Gill, J.B. Dixit "Digital Design and Computer Organisation"

[9] Histoire universelle des chiffres, Georges Ifrah, Robert Laffont, 1994 (Also:The Universal History of Numbers: From prehistory to the invention of the computer, Georges Ifrah, ISBN 0471393401, John Wiley and Sons Inc., New York, 2000. Translated from the French by David Bellos, E.F. Harding, Sophie Wood and Ian Monk)

[10]Kostopoulos, G. K., Digital Engneering. New York: John Wiley \& sons, Inc., 1975.

[11]FLORES, I., The Logic of Computer Arithmetic.Englewood Chffs, N. J.: Prentice-Hall, Inc. 1963.

[12]Behrouz A. Forouzan Data Communication And Networking 2nd edition Updated 